Originalveröffentlichung in: H. Roeder (Hrsg.), Das Erzählen in frühen Hochkulturen I. Der Fall Ägypten,

München 2009, S. 221-236

\title{
Die Piye(Pianchi)Stele: Erzählung als Medium politischer Repräsentation
}

\author{
Jan Assmann
}

Jede Behandlung politischer Narrativik im Alten Ägypten muss das eigentümliche Fehlen großer literarischer Erzählungen in Rechnung stellen, die hier in Entsprechung etwa zum Enuma Elish und zum Gilgamesh-Epos in Mesopotamien, zu den homerischen Epen in Griechenland oder zu den Vätererzählungen, Exodusüberlieferungen und Königsbiographien in Israel die Funktionen politischer Mythomotorik und kultureller Identitätsfundierung ausüben könnten. Ich will diese letztgenannten Begriffe kurz erläutern. Unter politischer Mythomotorik fasse ich Mythen zusammen, in denen eine Gruppe sich im Medium der Erzählung ihrer Herkunft und Ziele, ihrer fundamentalen Werte sowie ihrer Eigenart und Bedeutung vergewissert. ${ }^{1}$ Der Begriff der kulturellen Identität geht noch etwas über das Politische und Geschichtliche hinaus und bezieht sich auf fundamentale Vorstellungen und Normen von Sprache, Religion, Kunst und Moral, in denen eine Gruppe das Zentrum ihrer hochverbindlichen Überlieferungen erblickt. ${ }^{2}$ Solche normativen und formativen Überlieferungen politischer Mythomotorik und kultureller Identität hat es natürlich auch im Alten Ägypten gegeben. Zumindest was die politischen Erzählungen angeht, scheinen sie jedoch nicht die Ebene der Literatur erreicht zu haben. Natürlich müssen wir immer die ungeheuren Lücken unserer Überlieferung in Rechnung stellen und mit verallgemeinernden Aussagen und Schlüssen e silentio vorsichtig sein. Natürlich könnte es sein, dass uns die entsprechenden Erzählungen einfach verloren gegangen sind. Es ist aber auch mit der Möglichkeit zu rechnen, dass hier eine wirkliche Leerstelle vorliegt. Diese Annahme liegt meinen folgenden Überlegungen zugrunde. Sie würde den Ehrgeiz erklären, mit dem immer wieder einzelne Könige wie etwa Kamose oder Ramses II. versucht haben, der narrativen Verherrlichung ihrer Taten die große literarische Form zu geben und damit in diese Lücke vorzustoßen. Kamose hat auf zwei Stelen, die er im Amuntempel von Karnak aufstellen ließ, die Geschichte seines Befreiungskampfes gegen die Hyksos verewigt; ${ }^{3}$ der Anfang dieses Textes hat sich auf einer hieratisch beschrifteten Schreibtafel späterer Zeit erhalten, was zeigt, dass er damit in die literarische Zirkulation einging. ${ }^{4}$ Ramses II. hat die Schlacht bei Kadesch in zwei Formen monumentalisiert, als Bericht, also die übliche historische Inschrift, mit

1 Zu diesem Begriff s. Assmann, J., Frühe Formen politischer Mythomotorik. Fundierende, kontrapräsentische und revolutionäre Mythen, in: D. Harth/J. Assmann (Hgg.), Revolution und Mythos (1992), 39-61.

2 s. Ders, Das kulturelle Gedächtnis. Schrift, Erinnerung und politische Identität in frühen Hochkulturen (1992).

3 Habachi, L., The Second Stela of Kamose and His Struggle Against the Hyksos Ruler and his Capital (1972).

4 Gardiner, A. H., The Defeat of the Hyksos by Kamose. The Carnavon Tablet, in: Journal of Egytian Archaeology 3, 1916, 95-110. 
einer sehr groß angelegten landkartenartigen Darstellung, und als Gedicht. Das Gedicht ist auch auf Papyrus erhalten und war offensichtlich dazu bestimmt, als Literaturwerk zu zirkulieren. ${ }^{5}$

Unter diesen Versuchen ragt nun die Siegesstele, die der zweite König der 25. Dynastie, dessen Namen früher Pianchi und heute meist Piye gelesen wird, nach seinem Ägyptenfeldzug im Jahre 725 im Amuntempel von Gebel el-Barkal aufstellen ließ, mit großem Abstand hervor. ${ }^{6}$ Ein längerer und literarisch anspruchsvollerer Text ist im Alten Ägypten nie auf einer Stele verewigt worden. Dieser Text kommt vielleicht am nächsten an das heran, was man sich in Ägypten unter einem Epos vorstellen könnte. Denn auch das, eine Heldenepik, gibt es in Ägypten nicht. Jedenfalls scheint mir die Ausnahmestellung dieses Textes Grund genug, sich in einem Kolloquium über das Erzählen näher mit ihm zu beschäftigen. Erzählen - vielleicht sollte ich auch diesen Begriff versuchsweise kurz definieren. Erzählen ist die im Allgemeinen sprachliche, in selteneren Fällen auch bildliche Wiedergabe eines Stoffes, der eine bestimmte Struktur aufweist: die Struktur eines Vorgangs, Ereignisses oder auch einer Handlung, die in Raum und Zeit verankert ist und, um mit Aristoteles zu reden, Anfang, Mitte und Ende hat, wobei sich Anfang und Ende in aller Regel invers entsprechen. Das Ende stellt die Umkehrung des Anfangs dar: Das Problem des Anfangs, das die Handlung ins Rollen bringt, ist am Ende gelöst, die Krise behoben, das Fehlende restituiert, das Ziel erreicht. Natürlich gibt es Ausnahmen, die sich nicht in dieses Schema fügen, aber es ist in jedem Falle sinnvoll, sie zunächst einmal auf dieses Schema hin zu analysieren. Ich unterscheide hier nicht mit Harald Weinrich zwischen Berichten und Erzählen, also zwischen der berichtenden Wiedergabe eines Ereignisses, die dem Hörer Konsequenzen abverlangt, und der erzählenden Wiedergabe, bei der er sich entspannt zurücklehnen kann, ${ }^{7}$ sondern verwende einen beides umfassenden Begriff von Erzählen, der dem Beschreiben als seinem eigentlichen Gegensatz gegenübersteht. Unter Beschreiben verstehe ich die Wiedergabe eines Stoffes, dem die zeitliche Struktur fehlt. Natürlich geht in eine konkrete Erzählung mehr oder weniger viel Beschreibung ein. Daher muss man zwischen Erzählung als literarische Form und Erzählen als Sprechakt unterscheiden.

Für die Untersuchung ägyptischer Königsinschriften empfiehlt sich nun diese Unterscheidung ganz besonders, denn diese Texte pflegen in der Regel aus einem beschreibenden und einem erzählenden Teil zu bestehen. Der beschreibende Teil bezieht sich auf die Wesenszüge des Pharao, der erzählende auf ein bestimmtes Ereignis, die Stiftung eines Tempels, Festes oder Opfers, die Anlage eines Brunnens oder die Durchführung eines Feldzugs und vieles andere mehr. Den beschreibenden Teil

5 WaY, T. v. d., Die Textüberlieferung Ramses' II. zur Qades-Schlacht. Analyse und Struktur (1984); Assmann, J., Ägypten. Eine Sinngeschichte (1996), 278-301.

6 Grimal, N.-C., La stèle triomphale de Pi('ankh)y au Musée du Caire: JE 48862 et 47086-47089 (1981), siehe hierzu Goedicke, H., Pi(ankh)y in Egypt. A Study of the Pi(ankh)y Stela (1998) und vor allem TöröK, L., The Image of the Ordered World in Ancient Nubian Art. The Construction of the Kushite Mind, 800BC-300AD (2002), 368-398.

7 Weinrich, H., Tempus - Besprochene und erzählte Welt (1964). 
nennen wir „Eulogie“. ${ }^{8}$ Er besteht überwiegend aus einer Aneinanderreihung von Epitheta, also Nominalsyntagmen, die ohne jeden Zeitbezug dem König bestimmte Eigenschaften und Wesensmerkmale z. B. seiner göttlichen Abstammung, seiner alles überragenden Tapferkeit und Großzügigkeit, seiner unermüdlichen Tätigkeit für die Götter, den Staat, das Volk usw. prädizieren. Eulogie und Narration sind also die beiden wichtigsten Formen königlicher Repräsentation im Medium der Inschrift. Die meisten Königsinschriften streben ein ausgewogenes Verhältnis zwischen Eulogie und Erzählung an. Es gibt aber Inschriften, vor allem unter Ramses II., die nur aus Eulogie bestehen.' Umso auffallender ist in dieser Hinsicht wiederum der Fall der Piye-Stele: Sie besteht fast nur aus Erzählung.

Bevor wir uns dem Text selbst zuwenden, gilt es, sich in aller Kürze die historischen Rahmenbedingungen zu vergegenwärtigen. Im Süden Ägyptens, im Bereich des heutigen Sudan, hatte sich in der Gegend des vierten Katarakts im Laufe des 9. Jhs. v. Chr. das Reich von Napata gebildet, das den Nichtägyptologen vielleicht aus Verdis Aida bekannt ist. Im Neuen Reich, zwischen 1500 und 1100 v. Chr., hatte sich die ägyptische Herrschaft einmal bis in diesen Bereich erstreckt, aber das gehörte lange der Vergangenheit an, und die frühesten archäologischen Funde des Napata-Reiches zeigen, dass hier von ägyptischer Kultur nicht mehr die Rede sein konnte. Das ändert sich aber drastisch im Lauf des 9. Jhs. Das neue Herrscherhaus, das später als 25. Dynastie den ägyptischen Thron besteigen wird, befolgt eine Politik konsequenter Re-ägyptianisierung. ${ }^{10}$ Sein Aufstieg vollzog sich in drei Etappen. Der erste Schritt, der Aufbau einer lokalen Herrschaft am vierten Katarakt, liegt für uns im Dunkel und lässt sich nur an archäologischen Spuren ablesen. Der zweite Schritt ist die offenbar friedlich-freundliche Übernahme des thebanischen Gottesstaats und der Anspruch auf ägyptische Anerkennung als gleichrangiges Königtum. In dieser Zeit regierten in Ägypten mehrere Dynastien gleichzeitig, und die Kuschiten stellten sich auf eine Stufe mit den ägyptischen Dynastien und Fürstentümern. Der dritte Schritt ist dann die Überwindung dieser Polyarchie und die Restitution der pharaonischen Zentralherrschaft unter kuschitischer Führung.

Piye, der dritte König dieser Dynastie, strebt eine eigene Lösung an, die sich zwischen dem zweiten und dem dritten Schritt situiert. Er will die historisch gewachsene Struktur der Polyarchie nicht aufösen, sondern erhalten, sich aber als Großkönig über die in ihren Königs-, Fürsten- und Häuptlingstümern bestätigten und nur zu abhängigen Vasallen herabgestuften Lokalherrscher setzen. Er sieht offenbar einen besonderen Glanz und Stolz darin, nicht nur über ein Volk, sondern über Könige zu herrschen. Das ist eine im Rahmen der ägyptischen Tradition höchst originelle, offenbar mehr an neuassyrischen Vorbildern orientierte Staatsidee, und es ist

Assmann, J., s. v. „Eulogie“, in: Lexikon der Ägyptologie II, 40-46.

"Rhetorical Stela“, z.B. Doppelstelen C20 u. C22, Abu Simbel, KRI II, 315.08-321.06 (99 A, B).

Töröк, L., The Birth of an African Kingdom, Kush and Her Myth of the State in the First Millennium BC (1995); Ders., The Kingdom of Kush. Handbook of the Napatean-Meoritic Civilization (1997); Kendall, T., The Origin of the Napatan State: El Kurru and the Evidence for the Royal Ancestors, in: Meroitica 15, 1999, 3-177. 
möglicherweise die Neuheit dieser Idee, die hinter der einzigartigen Großartigkeit des Textes steht, den wir nun betrachten wollen. ${ }^{11}$

Die Siegesstele des Piye wurde 1862 am Gebel Barkal von einem ägyptischen Offizier auf Heimaturlaub gefunden und alsbald von Auguste Mariette, dem damaligen Generaldirektor der ägyptischen Altertümer, in sein Museum von Boulaq verbracht. Wenn ich oben Verdis Aida erwähnte, so ist das keine willkürliche Assoziation. Mariette widmete sich in den folgenden Jahren nicht nur dem Studium und der Publikation dieses Denkmals, sondern auch dem Exposé für ein Libretto, das er für die Einweihung des Suez-Kanals von dem berühmtesten Opernkomponisten der damaligen Zeit, Giuseppe Verdi, vertonen lassen wollte. ${ }^{12}$ Dabei hat er sich offensichtlich von dem neugefundenen Text anregen lassen. Denn auch in der Oper Aida geht es um eine von Napata ausgehende militärische Aktion. Allerdings werden die Vorgänge hier aus der Sicht des ägyptischen Herrschers, also als Rebellion, geschildert, und weil es sich um eine Oper handelt, steht nicht die politische Geschichte, sondern die Liebe im Vordergrund. Aufgrund verschiedener Umstände, unter denen auch der deutsch-französische Krieg von 1870/71 eine Rolle spielt, kam die Aufführung von Aida in Kairo erst im Dezember 1871 zustande. Dass Mariette sich von diesem Text zu einem Opernlibretto anregen lassen konnte, scheint mir ganz aufschlussreich. Mich erinnerte er, als ich ihn vor über zehn Jahren für meine ägyptische "Sinngeschichte“ genauer studierte, an ein Oratorium mit Rezitativen, Arien und Chören. Es ist ein Text, der die Rahmenbedingungen seiner Gattung, der Königsinschrift, sprengt und darin gleichsam unter Wert verkauft wird.

Die Rahmenbedingungen der Gattung sind Monumentalisierung und Publikation. Ein bestimmter Inhalt, der sich zu wechselnden Anteilen aus der Wesenscharakteristik eines Pharao und der Erzählung einer seiner Taten zusammensetzt, soll einerseits in dauerhafter Form aufgezeichnet an einem heiligen Ort für immer präsent gehalten, und andererseits einer Öffentlichkeit aus Götterwelt, Mitwelt und Nachwelt mitgeteilt werden. Dieser Inhalt hat, soweit er die Taten Pharaos betrifft, etwas mit unserem Begriff von Geschichte zu tun; jedoch wäre die Einordnung der Gattung Königsinschrift unter die Formen von Geschichtsschreibung ein Missverständnis. Es geht nicht um das Schreiben von Geschichte, in welchem Sinne auch immer, sondern um politische Repräsentation. Hinter diesen Texten und Bildern steht als assertierende Instanz nicht ein Historiker, sondern ein Herrscher. ${ }^{13}$ Die Ereignisse werden nicht um ihrer selbst willen geschildert, sondern als Manifestationen herrscherlicher Macht und Größe. Daher steht das Erzählen hier auch nicht im Dienst der Historie, im Sinne von Hayden White, sondern im Dienst der Identitätspräsentation. ${ }^{14}$

11 Vgl. Assmann, J., Sinngeschichte, 356-370.

12 Ders., Erinnertes Ägypten. Pharaonische Motive in der europäischen Religions- und Geistesgeschichte (2006), 153-184.

13 Bei ägyptischen Inschriften kommt es nicht auf den Verfasser an, sondern auf den Stifter bzw. Veranlasser der Inschrift, der für ihren Inhalt einsteht.

14 White, H. V., Metahistory: The Historical Imagination in Nineteenth Century Europe (1973), dt. Metahistory: die historische Einbildungskraft im 19. Jahrhundert in Europa (1991). Zum Begriff der Identitätspräsentation s. LüвBE, H., Zur Identitätspräsentationsfunktion der Historie, in: O. 
Sieht man einmal von der kommunikativen Funktion der Schrift, also Briefen, Nachrichten, Depeschen usw. ab, dann dient die Schrift in Ägypten drei Funktionen, die man Speicherung, Monumentalisierung oder Verewigung und Publikation oder Verbreitung nennen kann. Speicherung heißt: Etwas wird aufgezeichnet zum Wiedergebrauch. So werden Akten angelegt zur Beurkundung oder auch Wiedervorlage, Texte niedergeschrieben zur kultischen oder sonstigen Rezitation, oder auch Listen, Rezepte, Gesetze und sonstige wichtige Daten zur fallweisen Anwendung im Rechts-, Geschäfts- und Verwaltungsleben schriftlich niedergelegt. Diesen Formen von Speicherung dienen ca. 90 \% der altägyptischen schriftlichen Textproduktion, von denen uns allerdings nicht mehr als ein Tausendstel erhalten geblieben sein dürfte. Die restlichen grobgeschätzten $10 \%$ verteilen sich auf Monumentalisierung und Publikation. Monumentalisierung impliziert nicht notwendigerweise Publikation; eine Fülle von Inschriften wurde an für das Publikum unzugänglichen Orten aufgezeichnet. Wir dürfen aber nicht vergessen, dass die Ägypter in einer Welt lebten, in denen auch die Götter und die Toten zu der von ihnen adressierten Öffentlichkeit gehörten. Trotzdem ist es sinnvoll, den Begriff der Publikation solchen Texten vorzubehalten, die dazu bestimmt waren, von weiteren Teilen der Bevölkerung gelesen oder durch Zuhören rezipiert zu werden, das heißt: kulturell zu zirkulieren. ${ }^{15}$ Kulturelle Zirkulation konnte durch Inschriften an öffentlich besuchten Orten, z. B. Tempelvorhöfen, erreicht werden; ein wesentlich wirkungsvolleres Medium aber war die transportable Handschrift, die Papyrusrolle, und das allerwirkungsvollste Medium war der „kulturelle Text“, der zum Auswendiglernen im Unterricht bestimmt war, denn er erfüllte zugleich mit der Funktion der kulturellen Zirkulation auch die der Verewigung, weil er seinen Autor unsterblich machte. ${ }^{16}$ Hierzu gibt es einen berühmten Abschnitt in einer Weisheitslehre des 13. Jhs. v. Chr., der das Verfassen eines Weisheitsbuchs weit über das Errichten von Pyramiden und monumentalen Gräbern stellt und damit das horazische „Exegi monumentum aere perennius" um mehr als ein Jahrtausend vorwegnimmt. ${ }^{17}$

Die klassische Monumentalform für eine ägyptische Königsinschrift ist die oben abgerundete Stele mit einem halbkreisförmigen Giebelfeld über waagerechten Schriftzeilen. Im Giebelfeld ist eine Szene dargestellt, die sich in der Regel auf den eulogisch-zeitlosen, nicht den narrativ-ereignishaften Teil der Botschaft bezieht und den König vor der Gottheit darstellt, in deren Tempel die Stele aufgestellt ist.

Marquard/K. H. Stierle (Hg.), Identität (1979), 277-292.

$15 \mathrm{Zu}$ Texten, die aufgrund ihrer Anbringung (z. B. in Königsgräbern) jeder Zirkulation entzogen waren und daher als geheim gelten müssen, vgl. Baines, J., Restricted Knowledge, Hierarchy, and Decorum: Modern Perceptions and Ancient Institutions, in: Journal of the American Research Center in Egypt 27, 1990, 1-23, sowie Assmann, J., Ägyptische Geheimnisse (2004), Kap. 7 und 8.

16 Assmann, J., Cultural and Literary Texts, in: G. Moers (Hg.), Definitely Egyptian Literature. Proceedings of the Symposion "Ancient Egyptian Literature - History and Forms" (1999), 1-15; Ders., Kulturelle und literarische Texte, in: A. Loprieno (Hg.), Ancient Egyptian Literature. History and Forms (1996), 59-82.

17 Pap. Chester Beatty IV vso 2,5-3,11, s. Brunner, H., Ägptische Erziehung (1957), 177f.; Assmann, J., Schrift, Tod und Identität, in Ders., Stein und Zeit (1991), 173-175. 
Hier ist in aller Regel eine kultische Szene dargestellt, in der der König der Gottheit opfert. Manchmal überreicht auch eine Gottheit dem König ein Schwert. In jedem Falle aber bezieht sich die Darstellung nicht auf ein ort- und zeitkonkretes Ereignis, sondern auf eine zeitlose Konstellation. Die Piye-Stele fällt schon mit ihrer Giebelfeld-Darstellung vollkommen aus dieser Tradition heraus. Hier ist die Quintessenz der von Piye angestrebten politischen Ordnung ins Bild gesetzt: die Huldigung der verschiedenen von Piye unterworfenen, aber letztlich nur in ihren Titeln und Herrschaftsansprüchen bestätigten und bestärkten Königen und Fürsten. Unter diesen fehlt nur einer, der wichtigste, Tefnakhte, von dem im Folgenden noch viel die Rede sein wird. Vermutlich will Piye ihm die Aufnahme in diese ikonische Kodifizierung der Ordnung verweigern. Dargestellt ist in der Mitte Piye, nach rechts gewendet; hinter ihm thront in gleicher Blickrichtung Amun, von Mut (stehend) begleitet. Zu beiden Seiten dieser Zentralgruppe sind in je zwei Registern die unter- und mittelägyptischen Machthaber dargestellt. Die rechte Gruppe, der Piye sich zuwendet, wird angeführt im oberen Register vom Königspaar von Hermupolis. Diese beiden Figuren sind durch aufrechte Haltung deutlich herausgehoben. Die anderen acht sind in Proskynese dargestellt. Die rechte Gruppe umfasst ausschließlich königliche Personen, als solche kenntlich gemacht nicht nur durch Titel und Kartuschenschreibung, sondern auch durch das Uräusdiadem. Die linke Gruppe stellt vier libysche Stammeshäuptlinge, kenntlich an ihrer Feder, dar und den Prinzen Pediese. Entscheidend ist, dass diese Personen nicht als „Rebellen“ und „elende Feinde“, sondern im vollen Ornat ihrer herrschaftlichen Identität erscheinen. Damit wird ein politischer Sachverhalt, der jeder traditionellen Ideologie krass widerspricht, auch denen, die nicht lesen können, bildlich vor Augen geführt. Weit entfernt, in diesen Zuständen Symptome von Chaos und Anarchie zu erkennen, affirmiert Piye sie als gottgewollte Ordnung, indem er sie im Bildfeld seiner Stele ikonisch kodifiziert.

Für den erzählenden Teil eines Feldzugberichts gibt es seit der frühen 18. Dynastie ein Schema, wie ja auch schon diesen Feldzügen selbst eine Art traditionelles Drehbuch zugrunde gelegen haben dürfte. Dieses Schema sieht die mit der Partikel jst eingeleitete Schilderung einer Situation vor, in der sich der König gerade befindet. Diese Partikel dient als Signal, dass jetzt von Eulogie auf Erzählung umgestiegen wird. In diese Situation platzt ein Bote mit der Nachricht, dass in einem unterworfenen Fremdland eine Rebellion ausgebrochen ist. Daraufhin gerät der König in Zorn, schwört einen Eid bei seinem Vater Amon, was der folgenden Aktion die göttliche Legitimation sichert, und bricht zum Feldzug auf, der dann mit Sieg und Heimkehr endet. Piye zitiert gelegentlich das traditionelle Schema, aber nur um damit deutlich zu machen, dass er nicht diesem, sondern einem völlig neuen Drehbuch folgt.

Der Text beginnt mit der Datierung (Jahr 21 unter König Piye $=728$ v. Chr.) und legt die eulogische Exposition, auf einige wenige Verse verkürzt, dem König als eröffnende direkte Rede selbst in den Mund:

Befehl, den Seine Majestät ausgesprochen hat:

"Hört, was ich getan habe in Überbietung der Vorfahren: 

Ich bin ein König, Symbol Gottes,
lebendes Abbild des Atum,
der aus dem Leib hervorging, zum Herrscher bestimmt,
so dass sich vor ibm fürchteten, die älter waren als er.
$<$ Sein> Vater wusste, seine Mutter erkannte:
er ist schon im Ei zum Herrscher bestimmt.
Der präsente Gott, den die Götter lieben,
Sohn des Re, der mit seinen Händen handelt,
Miamana Piye."

Dann übernimmt bereits der epische Erzähler mit dem traditionellen Botenauftritt:

Man kam, S. M. zu melden:

Es gibt einen Häuptling des Westens,

der Graf und Fürst von Sais, Tefnacht,

der ... hat den ganzen Westen in Besitz genommen,

von den Nordsümpfen bis Lischt,

und fährt jetzt siidwärts mit einem großen Heer,

das ganze Land verbündet in seinem Gefolge, usw. usw.

Damit haben wir, erzähltechnisch gesprochen, die Ausgangssituation: die Krise, die behoben werden muss. Sie wird nicht als Rebellion geschildert, sondern als eine vom Fürsten von Sais ausgehende Einigungsbewegung mit dem Ziel, die „Polyarchie" der 3. Zwischenzeit zu beenden und eine neue Form politischer Einheit unter seiner Oberherrschaft aufzubauen. An diesem Punkt setzt die Handlung ein, die in der Piye-Stele berichtet wird. Piye, anstatt wie es das traditionelle Schema vorschreibt, bei diesen Botschaften in rasenden Zorn auszubrechen, hört sie mit Freude und quittiert sie mit herzlichem Lachen. Die zweite Botschaft kommt von den bedrängten "Grafen und Garnisonskommandanten in ihren Städten“. Sie melden, dass nun auch Nimlot in Hermupolis unter großen Druck geraten und zu Tefnachte übergelaufen ist.

Darauf schickt Piye zu den Kommandanten der in Ägypten stationierten Truppen den Befehl, Tefnachte anzugreifen, und schickt überdies ein Heer nach Ägypten aus, das er sorgfältig instruiert. Keine nächtlichen Überfälle, Kampf nur bei Tage und nur nach vorheriger Ankündigung; wenn der Gegner Aufschub wünscht, soll er gewährt, wenn er Verstärkung abwarten will, soll gewartet werden. Gerade gegen diese Verstärkungstruppen soll gekämpft werden. Ihnen soll der Kampf folgendermaßen angekündigt werden:

Wir wissen (zwar) nicht, wen wir anreden sollen, wenn wir uns an das Heer wenden,

(aber höre:) Spann den besten Streitwagen deines Stalls an und ordne die Schlachtreihen!

Dann wirst du erfahren, dass es der Gott Amun ist, der uns gesandt hat!

Piye will seinem Krieg ein ganz besonderes und, wie mir scheint, völlig neuartiges Kriegsrecht zugrunde legen, um ihn ostentativ als einen gerechten Krieg zu führen, ja mehr noch, als einen heiligen Krieg, denn seine strenge Selbstbeschränkung 
speist sich aus der Gewissheit göttlichen Auftrags. ${ }^{18}$ Das ist das neue Drehbuch, das er seinen Aktionen und deren narrativer Repräsentation zugrunde legt.

Während der erste Teil der Instruktionsrede die Regeln der Kriegsführung betrifft, bezieht sich der zweite auf das Verhalten des Heeres in Theben. Theben erscheint hier als eine heilige Stadt, in der strengster Friede gewahrt werden muss. Das Heer soll die Waffen niederlegen, sich im Wasser reinigen und priesterliches Leinen anziehen. Vor Gott ist militärische Stärke sinnlos:

Kein Held hat Kraft ohne ihn.

Er macht Schwache zu Starken, so dass Viele vor Wenigen davonlaufen und Einer allein Tausende besiegt.

Daher sollen die Soldaten sich reinigen, vor ihm niederfallen und ihn anrufen:

Weise du uns den Weg, auf dass wir im Schatten deines Schwertarms kämpfen.

Die Truppe, die du ausgesandt hast, deren Angriff gelingt, und vor ihr wird die Menge von Schrecken ergriffen.

Die Maßnahmen, die hier geschildert werden, sind absolut neuartig. Wo hätte es in Ägypten jemals Vergleichbares gegeben? Ein ganzes Heer soll sich priesterlich reinigen und kleiden, um in den Tempel eintreten und Amun um Beistand bitten zu können. Es handelt sich um ein regelrechtes Ritual der Kriegseröffnung.

Das von Piye ausgesandte Heer zieht über Theben nach Herakleopolis und stößt dort auf die Koalition von drei Königen und fünf Fürsten, die sich mit ihren Armeen dem Tefnachte angeschlossen haben. Es kommt zu zwei Kämpfen, in deren Folge sich das Heer der Koalierten nach Unterägypten zurückzieht, während Nimlot nach Hermupolis, seinem Stammsitz entkommt. Daraufhin belagert das kuschitische Heer die Stadt Hermupolis und erstattet dem König Meldung.

Jetzt erst, bei diesen Siegesmeldungen, gerät der König in seinen vom traditionellen Drehbuch vorgeschriebenen Zorn. Er „ergrimmt wie ein Panther" darüber, dass die Koalierten entkommen sind, schwört den traditionellen Schwur, der ihn des göttlichen Beistands versichert, und bricht selbst nach Ägypten auf, will aber vorher (was wiederum etwas völlig Neues ist) in Theben das Luxorfest in aller vorgeschriebenen Weise begehen, was immerhin ca. drei Wochen in Anspruch nimmt. Das Heer, das vom Zorn Seiner Majestät erfahren hat, versucht durch eine Reihe erfolgreicher Aktionen seinen Herrn zu besänftigen: Dreimal heißt es „da erstatteten sie S. M. Meldung, aber sein Herz war darüber nicht zufrieden". Nach Abschluss der thebanischen Festlichkeiten begibt sich Piye zum belagerten Hermupolis, hält seinem Heer eine Scheltrede und verschärft die Belagerung, bis Hermupolis anfängt, „Verwesungsgeruch auszuströmen“. Der in die Knie gezwungene König Nim-

18 Kang, S.-M., Divine War in the Old Testament and in the Ancient Near East (1989); WAY, T. v. d., Göttergericht und „heiliger Krieg“ im Alten Ägypten. Die Inschriften des Merenptah zum Libyerkrieg des Jahres 5 (1992). 
lot schickt Gesandte, schließlich seine Königin. Leider sind große Abschnitte des Folgenden zerstört. Aber nach einer langen Lücke gehen die Reden weiter: Offenbar bekehrt sich Nimlot und kehrt reumütig in sein Vasallenverhältnis zu Piye zurück. Die Vorgänge um Hermupolis enden mit der Unterwerfung des Königs, der Konfiskation seines Schatzes, einem Dankopfer für Thot, den Gott von Hermupolis, und einer Szene höchst besonderer Art, die einen zweiten Wutanfall des Piye auslöst. Der Text berichtet im ganzen von drei solchen Zornesausbrüchen, und alle äußern sie sich in einem feierlichen Schwur des Königs. Was ist passiert? Bei der Inspektion des Pferdestalls stellt Piye fest, dass (die?/einige?) Pferde verhungert sind; was Wunder bei einer monatelang belagerten Stadt. Aber wie „liest" Piye diesen Befund?

So wahr ich lebe und Re mich liebt

und meine Nase sich mit Leben verjüngt:

Das hier ist in meinen Augen schlimmer, meine (!) Pferde verhungern zu lassen, als alle deine sonstigen Verbrechen.

Er nimmt diesen Vorfall zum Anlass, seine göttliche Sendung deutlicher als irgendwo sonst in dem langen Text zu proklamieren:

Weißt du nicht, dass der Schatten Gottes auf mir liegt?

Er lässt meine Sache nicht scheitern.

Wenn ein anderer das getan hätte, der mich nicht kennt,

den hätte ich nicht getadelt deswegen.

Ich bin im Leibe geboren, geschaffen im göttlichen $E i$,

der Same Gottes ist in mir.

So wahr sein Ka dauert, ich handle nicht ohne ihn,

er ist es, der mir befiehlt was ich tue.

Piye - natürlich nicht als Verfasser, aber als „Assertor" dieses Textes - gibt sich alle Mühe, die dogmatischen Rahmenbedingungen seines königlichen Handelns nicht in der traditionellen Form der Eulogie der Erzählung voranzustellen, sondern aus der Erzählung selbst hervorgehen zu lassen. Das ist das Prinzip der Heldenepik. Der Held konstituiert sich als solcher und gewinnt seine heroische Identität aus seinen Taten und Handlungen, die das Thema der Erzählung bilden, und nicht aus zeitlosen Wesenseigenschaften. Eine heroische ist immer eine erzählte Identität. ${ }^{19}$ Der Sinn dieser Szene ist, Nimlots Schuld an den Folgen der Belagerung zu definieren. Wer seine Tore wider besseres Wissen vor dem gottgesandten Herrscher verschließt, trägt die Schuld an allen Folgen der dadurch erzwungenen Belagerung. Diese Schuld hat bei ihm den Charakter einer Sünde, im Unterschied zu einem Feind, der in Notwehr handelt.

Die Einnahme von Hermupolis verfehlt ihren Eindruck nicht: Vier weitere Fürstentümer bieten ihre Unterwerfung an. Piye schont die Zivilbevölkerung und beschränkt sich darauf, die Schatzhäuser zu konfiszieren, die Magazine dem Amuntempel in Karnak zu überschreiben und ein Dankopfer für die Götter der Stadt

19 Vgl. Neumann, M., (Hg.), Erzäblte Identitäten (2000). 
zu veranstalten. Diese Praxis passt in sein Rollenkonzept von pietas und zu seinem theokratischen Staatsbegriff.

Die Erzählung kommt nun zu ihrem Höhepunkt, der Einnahme von Memphis, die die Mitte und die Peripetie des Piye-Epos bildet. Hier fordert Piye die Öffnung der Tore, damit er den Göttern von Memphis opfern und weiter nach Norden ziehen kann, und verweist auf das Schicksal der südlichen Städte, denen kein Haar gekrümmt wurde ,außer den Rebellen, die Gott beleidigt haben “. Widerstand gegen den gottgesandten Herrscher ist Blasphemie. Memphis jedoch verschließt seine Tore und macht sogar einen Ausfall. Die Geschichte der Einnahme von Memphis vermag am besten die eigenwillige Erzählperspektive des Textes zu verdeutlichen. Der ganze Abschnitt nimmt ungefähr 170 Verse, also ein Fünftel des Gesamttexts ein. Davon entfallen allein 58 auf Reden:

1. das Ultimatum des Piye (15)

2. die Rede des Tefnacht (16), der die Stadt zum Durchhalten anfeuert

3. die Beratung im Heer des Piye über die Strategie der Belagerung (9)

4. der Schwur des Piye, der über diese Reden zum dritten Mal in Zorn gerät und unter Verweis auf den Willen Amuns auf sofortigen Angriff drängt: „Ich will Memphis wie ein Wolkenbruch einnehmen" (10)

5. die Anfeuerungsrede des Piye (8)

Die eigentlichen Kampfhandlungen werden nur kurz erwähnt. Viel wichtiger als der militärische Aspekt der Einnahme von Memphis ist dem König die Psychologie der Rebellion und Belagerung, der geforderten, verweigerten und schließlich erzwungenen Anerkennung, wie sie die Reden beleuchten, sowie dann die heiligen Handlungen in Memphis, Babylon (Cheraha) und Heliopolis (72 Verse). Piye beansprucht hier für sich nicht nur die persönliche Frömmigkeit seiner inneren Motivation, wie es zur traditionellen Königsrolle gehört, sondern darüberhinaus eine Art liturgischer Kompetenz, wie sie den berufenen und befugten professionellen Priester kennzeichnet, der das Ritual kennt und auf kultische Reinheit achtet. Das Siegel lösen, den Riegel öffnen, den Gott schauen, den Riegel schließen und das Siegel anlegen sind Handlungen, die zwar in königlichem Auftrag, aber immer von einem professionellen Priester vollzogen werden. Ein König mischt sich hier nicht ein. Piye verrichtet in Heliopolis regelrechte Priesterdienste und macht damit die Neuartigkeit des scripts, dem er folgt, noch einmal besonders deutlich.

Den Schluss bilden die Huldigungen der sich unterwerfenden Deltafürsten, was wiederum in der Form langer Reden vor sich geht und mit einer Auflistung aller Machthaber schließt, die die Oberherrschaft des Piye anerkennen. Schließlich schickt auch Tefnachte selbst ein Friedensangebot, und seine Rede von nicht weniger als 39 Versen bildet den Höhepunkt ihrer Gattung. Damit ist die Mission des Piye erfüllt. Der Text schließt mit der Aufwartung, die die vier Könige unter den unterworfenen Machthabern dem Kuschiten in seinem Palast erweisen wollen. Hier ergibt sich nun ein Problem kultischer Reinheit, von dem vorher nie in vergleichbaren ägyptischen Inschriften die Rede war. Drei Könige, heißt es, müssen draußen warten, nur einer darf hinein. Außer Nimlot sind nämlich alle unbe- 
schnitten und essen Fisch, was „ein Abscheu des Palastes "ist. Wir erfahren hier zum ersten Mal von Reinheitsvorschriften, für die die Ägypter der Spätzeit bekannt waren. Piye stellt sich in dieser die Delta-Könige demütigenden Szene als ein nicht nur legitimer, sondern im priesterlichen Sinne „reiner“ König dar und spielt seine Reinheit gegen die Libyer aus. Hier werden mit den Mitteln religiöser Reinheitsvorschriften ganz neuartige Grenzen gezogen.

Die auffallendste Eigenschaft des Piye-Textes ist sein literarischer Charakter. Das ist zunächst schon einmal eine Sache des Umfangs. Mit seinen 900 Versen hat das Piye-Epos den Umfang (nach ägyptischen Proportionen) eines zweibändigen Romans. Ein Text auch nur des halben Umfangs ist nie in Ägypten auf eine Stele geschrieben worden. Man versteht diesen Text nicht vollständig, wenn man sich nicht klar macht, dass er damit den Rahmen einer Königsinschrift sprengt und etwas ganz anderes anstrebt, nämlich ein Königsepos, für das es in der ägyptischen Tradition kein Vorbild gibt (allenfalls das halb so umfangreiche Gedicht von der Kadesch-Schlacht käme hierfür in Betracht), auf das diese Tradition aber in gewisser Weise vorbereitet ist und sozusagen gewartet hat.

Piye überschreibt seinen Text zwar als „Dekret" ( $w \underline{d}$, das übliche Wort sowohl für "Dekret" als auch für "Stele"), aber es ist ein Buch, was ihm vorschwebt. Der Text gliedert sich in zwei Teile von annähernd gleicher Länge. Höhepunkt des ersten Teils ist die Einnahme von Hermupolis, mit Nimrot als Hauptgegner, Höhepunkt des zweiten die Einnahme von Memphis mit Tefnacht als Hauptgegner. Den Kämpfen des ersten Teils gehen die heiligen Handlungen in Theben voraus, den Kämpfen des zweiten folgen die heiligen Handlungen in Memphis, Babylon und Heliopolis.

Auch das Verhältnis von Erzählung und Rede - ungefähr 2:3 - entspricht dem Aufbau literarischer Erzählungen. ${ }^{20}$ Wir haben Eulogie und Erzählung gegenübergestellt und müssen nun innerhalb der Erzählung noch einmal unterscheiden zwischen der eigentlichen Erzählung und den eingeschobenen direkten Reden. Die Erzählung bezieht sich auf den Handlungsablauf, die Reden auf dessen lyrisch-subjektive "Innenbeleuchtung“. Die narrativen Partien verwenden die klassischen Formeln und Verbformen der mittelägyptischen Erzählung: „Als aber die Erde hell wurde, und der Morgen anbrach ... ", die feierliche, abschnittseröffnende Verbform „x war es, was er machte ... ", der "Narrativ“ " $c c^{c} . n s \underline{d} m=f$ "da hörte er", der die Erzählung einen Schritt vorantreibt, und das resümierende $w n . j n=f \underline{h r} s \underline{d m}$, das wir auch mit "da hörte er" wiedergeben und das aber einen vorangehenden Erzählabschnitt resümierend abschließt. Die narrativen Partien bestimmen den Gesamtaufbau des Textes, die Reden sind in sie eingebettet, auch wenn sie quantitativ überwiegen.

Mehr noch als in der schieren Größenordnung zeigt sich der literarische Charakter des Textes in seinen bewussten Rückgriffen auf große Traditionen der ägyptischen Literatur, seiner Verwendung sprachlicher, formaler und motivlicher Modelle, seinem „Klassizismus“. ${ }^{21}$ Piye mobilisiert in diesem Text das kulturelle Ge-

20 Vgl. Hintze, F., Untersuchungen zu Stil und Sprache neuägyptischer Erzählungen (1950).

${ }^{21}$ Vgl. Grimal, N.-C., Bibliothèques et propagande royale à l'époque éthiopienne, in: J. Vercoutter 
dächtnis Ägyptens und legitimiert sich durch „Bildung" mindestens ebenso wie durch Frömmigkeit (pietas) und militärischen Erfolg. ${ }^{22}$ Die Höhepunkte stellen die Reden dar, und unter diesen ragen wiederum die loyalistischen Bekenntnisse hervor. Die Reden des Nimlot, Peftjauawi-Bastet und ganz besonders des Tefnachte erinnern an die ramessidischen Gebete der Persönlichen Frömmigkeit mit ihren Bauformen der hymnischen Anrufung, Klage, Bekenntnis, Gelübde und Lobpreis. Peftjauawi-Bastet wendet sich mit folgenden Worten an Piye:

(Anrufung) Gegrïßet seist du, Horus, starker König,

Stier, der Stiere angreift!

(Klage) Die Unterwelt hat mich ergriffen,

ich bin versunken in Finsternis.

Gib mir das Licht Deines Angesichts!

Ich habe keinen Gefährten gefunden am Tage des Unglücks

keinen Beistand am Tage des Kampfes, ${ }^{23}$

nur dich allein, Du starker König -

decke auf die Finsternis in meinem Gesicht!

(Gelübde) Ich will dir dienen mit meiner Habe,

Herakleopolis soll deiner Behörde steuerpflichtig sein.

(Lobpreis) Du bist wahrlich Harachte, das Oberhaupt der Zirkumpolarsterne, solange er existiert, existierst du als König.

Geht er nicht unter, gehst du nicht unter,

König von Ober- und Unterägypten, Piye, der ewig lebt!

Noch wesentlich umfangreicher ist die Botschaft des Tefnachte:

(Anrufung und Klage) Sei gnädig! Ich kann dein Gesicht nicht sehen in den Tagen des Zorns (oder: der Blindheit).

Ich kann nicht bestehen vor deinem Gluthauch

und erschrecke vor deiner Hoheit.

Siehe, du bist Seth, der Erste des Südens,

Month, der starke Stier.

Zu welcher Stadt auch immer du dein Gesicht wendest,

du wirst mich nicht darin finden, und wenn ich bis ich zu den Inseln des Meeres fiehen müsste.

Ich fürchte mich vor deiner Zornesmacht

und sage mir: Seine Flamme ist mir feindlich.

Ist denn das Herz deiner Majestät nicht gekühlt durch das, was du mir angetan hast?

Ich bin doch wabrhaftig elend.

Strafe mich nicht nach meiner Sünde,

der du die Waage fübrst und mit dem Gewichtstein urteilst.

Lege mir dreifache Buße auf,

aber schone die Saat, damit du sie erntest zur Zeit,

reiße den Fruchtbaum nicht aus mit seiner Wurzel.

(Hg.), Livre du centenaire (1980), 37-48.

22 Auch diese Demonstration von klassischer literarischer Bildung ist ein Zug, der an die Ideologie des neoassyrischen Hofes erinnert. Vgl. dazu Maschinist, P., The Assyrians and their Babylonian Problem, in: Jahrbuch des Wissenschaftskollegs zu Berlin 1984/85, 1985, 353-364.

23 Diese beiden Verse sind Zitate aus der Lehre des Amenemhet I. 
So wahr dein Ka dauert, sitzt der Schrecken vor dir mir im Leibe,
steckt mir die Furcht vor dir in den Knochen.
Ich kann nicht im Bierhaus sitzen,
und lasse mir nicht Harfe spielen.
Ich esse das Brot des Hungers
und trinke das Wasser des Durstes
seit dem Tag, da du meinen Namen hörtest.
Schmerz sitzt mir im Gebein,
mein Kopf ist kahl, mein Kleid staubig,
bis, man' mir die Neith besänftigt.
Lang ist der Lauf, den du gegen mich genommen hast, dein Gesicht auf mich gerichtet.
Wird mein Ka einmal losgesprochen,
wird der Diener gereinigt von seinen Verfehlungen?

Die psychologische Innenbeleuchtung, die diese Reden dem Geschehen geben, zeigt uns die Haltung eines religiösen Loyalismus, der in dem gottgesandten König auch den persönlichen Heilbringer sieht und sich ihm mit ganzem Herzen und ganzem Vermögen anschließt. Der Text legt auf die Gestaltung dieser Reden größten Wert. Sie entfalten die semantische Dimension der Geschichte, indem sie die innere Motivation des Handelns aufdecken, Haltungen und Einstellungen ausleuchten, die Vorgänge deuten, Ziele nennen. Dieses Verhältnis von Rede und Erzählung, das ungefähr dem Verhältnis von Arien und Rezitativen in einem Oratorium oder einer opera seria entspricht, ist die Signatur des Literarischen in Ägypten. Hierin unterscheiden sich z. B. auch Bericht und Gedicht der Kadesch-Schlacht. Die Einschaltung langer direkter Reden mit subjektiver Beleuchtung des erzählten Geschehens wäre in einer privaten Grabinschrift, in der ja auch oft erzählt wird, undenkbar.

Ich war ausgegangen von dem anscheinenden Fehlen eines großen politischen Epos in Ägypten. Es gibt natürlich die großen Mythen, die auch eine politische Dimension haben, vor allem der Mythenkreis um Horus und Seth, ${ }^{26}$ aus dem dann in der Spätzeit der Horusmythos von Edfu als eine Art episches Festspiel

24 Was nur Piye als gottgesandter König tun kann.

25 Das Bild des büßenden Tefnachte, der sein Leben bei Wasser und Brot fristet und auf die Annehmlichkeiten des Harfenspiels verzichten muss, erinnert auffallend an eine Anekdote, die Plutarch und Diodor von diesem Herrscher erzählen. Es heißt da, er habe die ägyptische Hochkultur insgesamt verworfen und habe sich zu einem primitiven Lebensstil bekannt. Auf einem Feldzug gegen die Araber habe der König einmal ohne die übliche Ausrüstung auskommen und mit dem vorlieb nehmen müssen, was gerade zur Hand war. Er habe daran so großen Geschmack gefunden, dass er sich dem einfachen Leben verschrieben und Menes als den Begründer der ägyptischen Luxus-Kultur verflucht habe. Anschließend habe er den Fluch auf einer Stele aufzeichnen und im Amuntempel von Karnak aufstellen lassen (Plutarch, De Iside et Osiride, Kap. 8, hg. v. J. G. Griffiths (1970), 128, 282. Diodor, Bibl. Hist. I, 45. Yoyotтe, J., Notes et documents pour servir à l'histoire de Tanis I. Bocchoris à Tanis et l'expansion des premiers rois saïtes vers l'Orient, in: Kêmi 21, 1971, 40-42). Dieser Anekdote zufolge nimmt Tefnachte einen Lebensstil freiwillig auf sich, über den er sich in dem Piye-Epos beklagt; aber in beiden Quellen verbindet sich mit seinem Namen das Motiv außergewöhnlicher Entbehrungen.

26 Pap. Chester Beatty I, rto., dazu Verhoeven, U., Ein historischer „Sitz im Leben“ für die Erzählung von Horus und Seth des Papyrus Chester Beatty I, in: M. Schade-Busch (Hg.), Wege öffnen. Festschrift für Rolf Gundlach zum 65. Geburtstag (1996) 347-363. 
hervorgeht. ${ }^{27}$ Daneben gibt es eine Art mythischer Leerform, die sich entfernt dem alttestamentlichen Messianismus vergleichen lässt. Das ist der Mythos des Heilskönigs, der von Gott gesandt ist, um die Dinge auf Erden wieder in Ordnung zu bringen, das heißt, die Krise zu beheben, den Mangel zu beseitigen und nach längerer Leidenszeit eine Glücks- und Heilszeit heraufzuführen. ${ }^{28}$ Wir stoßen auf diesen Mythos zuerst in den Prophezeiungen des Neferti, der ihn für Amenemhet I. als den Liquidator der 1. Zwischenzeit und den eigentlichen Gründer des Mittleren Reichs in Anspruch nimmt, dann auf eine der Wundererzählungen des Papyrus Westcar, die ihn auf die ersten drei Könige der 5. Dynastie bezieht. ${ }^{29}$ Beide Texte gehören zur schönen Literatur. Unter der Königin Hatschepsut aber stoßen wir auf Texte, die zeigen, dass diese offenbar von einem besonderen Sendungsbewusstsein erfüllte Königin diesen Mythos im Medium der Königsinschrift auf sich beziehen und sich als die Liquidatorin der zweiten Zwischenzeit, Gründerin des Neuen Reichs und in diesem Sinne als gottgesandte Heilskönigin darstellen wollte. ${ }^{30} \mathrm{Ganz}$ so weit gehen Kamose, Ramses II. und Piye nicht, sie reden nicht von einer Leidenszeit, sondern nur von einer schweren Krise, die sie beheben, aber das Motiv der göttlichen Sendung steht bei ihnen ganz im Vordergrund. Kamose erwähnt es am Anfang und schreibt dann die folgenden Kampfhandlungen ganz auf das Konto seiner persönlichen Tapferkeit, Ramses II. aber schreibt seine Rettung aus höchster Not bei Qadesch allein dem persönlichen Eingreifen Amuns zu und Piye inszeniert sich in seiner Siegesstele, wie wir gesehen haben, durchweg als priesterlich-frommer Vollstrecker des göttlichen Willens. Von all diesen Herrschern hat es nur Ramses II. geschafft, wirklich zum Helden eines nationalen Epos zu werden, aber nur auf Kosten seiner historischen Identität, denn er verschmolz mit Sesostris I. und III. zum Helden des in griechisch-römischer Zeit zirkulierenden Sesostris-Romans. ${ }^{31}$

Worauf es mir in diesem Beitrag ankam, war, die Spannung zwischen der im Grunde außerliterarischen Gattung der Königsinschrift und der literarischen Form aufzuzeigen, wie sie sich mit ihren Vorgaben von Umfang und Aufbau, ihren Verfahren der Verteilung des Stoffs auf Reden und Erzählung, ihren spezifischen Sprachformen narrativer Darstellung und kühner Metaphern (wie das Uroboros-Bild für die Belagerung oder der Wolkenbruch für die Eroberung in Form des „Blitzkriegs"), den Techniken von Spannungsaufbau und der Herausarbeitung von Höhepunkten in ganz anderen Gebieten der ägyptischen Schriftkultur herausgebildet hat. Piye darf als der ägyptische König gelten, der sich diese literarische Form, die in einer Königsinschrift von Haus aus nichts zu suchen hat, am konsequentesten zu-

27 Farrman, H. W., The Triumph of Horus: An Ancient Egyptian Sacred Drama (1974).

28 ZANDEE, J., Le messie, in: Revue de l'histoire des religions 180, 1971,3-28; Assmann, J., Sinngeschichte, 418-430.

29 Posener, G., Littérature et politique dans l'Égypte de la XIIe dynastie (1956), 156f.; HeLcK, W., Die Prophezeiung des Neferti (1970); Papyrus Westcar, P.3033, Papyrus-Sammlung der Staatlichen Museen zu Berlin. Erman, A., Die Märchen des Papyrus Westcar (1890); Brunner-Traut, E., Altägyptische Märchen (1963), Nr.3, 19-24.

30 Assmann, J., Das Sendungsbewußtsein der Hatschepsut, in: G. Moers et al. (Hgg.), jn.t $\underline{d}$ r.w - Festschrift für Friedrich Junge (2006), 59-72.

31 LANGE, K., Sesostris. Ein ägyptischer König in Mythos, Geschichte und Kunst (1954). 
nutze gemacht hat. Das Rätsel, warum ausgerechnet ein Außenseiter wie Piye, Angehöriger eines von den Ägyptern traditionell besonders verachteten Fremdvolks, der Gattung der Königsinschrift und der Form der narrativen politischen Repräsentation einen so einzigartigen Höhepunkt verschafft, möchte ich als solches stehen lassen; mir kam es mehr darauf an, das Phänomen in seiner Besonderheit herauszustellen. Einerseits fühlte er sich als Außenseiter gewiss unter besonderem Legitimationsdruck, andererseits dürfte ihn vielleicht auch sein starkes Sendungsbewusstsein und die Neuartigkeit seiner politischen Vision, nämlich Ägypten nicht in traditioneller Weise als Pharao über Untertanen, sondern als Großkönig über Königen zu regieren, zu dieser einzigartigen Repräsentationsform beflügelt haben.

\section{Bibliographie}

Assmann, J., s. v. „Eulogie“, in: Lexikon der Ägyptologie II (1977), 40-46.

Ders., Schrift, Tod und Identität, in: Ders. (Hg.), Stein und Zeit (1991), 173-175.

Ders., Das kulturelle Gedächtnis. Schrift, Erinnerung und politische Identität in frühen Hochkulturen (1992).

Ders., Frühe Formen politischer Mythomotorik. Fundierende, kontrapräsentische und revolutionäre Mythen, in: D. Harth/J. Assmann (Hgg.), Revolution und Mythos (1992), 39-61.

Ders., Ägypten. Eine Sinngeschichte (1996).

Ders., Kulturelle und literarische Texte, in: A. Loprieno (Hg.), Ancient Egyptian Literature. History and Forms (1996), 59-82.

Ders., Cultural and Literary Texts, in: G. Moers (Hg.), Definitely: Egyptian Literature.

Proceedings of the Symposion "Ancient Egyptian Literature - History and Forms" (1999), 1-15.

Ders., Ägyptische Geheimnisse (2004).

Ders., Erinnertes Ägypten. Pharaonische Motive in der europäischen Religions- und Geistesgeschichte (2006).

Ders., Das Sendungsbewußtsein der Hatschepsut, in: G. Moers et al. (Hgg.), jn.t $\underline{d r}$ w. Festschrift für Friedrich Junge (2006), 59-72.

Baines, J., Restricted Knowledge, Hierarchy, and Decorum: Modern Perceptions and Ancient Institutions, in: Journal of the American Research Center in Egypt 27, 1990, 123.

Brunner, H., Ägyptische Erziehung (1957).

Brunner-Traut, E., Altägyptische Märchen (1963).

Erman, A., Die Märchen des Papyrus Westcar (1890).

Fairman, H. W., The Triumph of Horus: An Ancient Egyptian Sacred Drama (1974).

Gardiner, A. H., The Defeat of the Hyksos by Kamose. The CarnavonTablet, in: Journal of Egyptian Archaeology 3, 1916, 95-110.

Goedicke, H., Pi(ankh)y in Egypt. A Study of the Pi(ankh)y Stela (1998).

Grimal, N.-C., Bibliothèques et propagande royale à l'époque éthiopienne, in: J. Vercoutter (Hg.), Livre du centenaire (1980), 37-48.

Ders., La stèle triomphale de Pi('ankh)y au Musée du Caire: JE 48862 et 47086- 47089 (1981).

Habachi, L., The Second Stela of Kamose and His Struggle Against the Hyksos Ruler and His 
Capital (1972).

Helck, W., Die Prophezeiung des Neferti (1970).

Hintze, F., Untersuchungen zu Stil und Sprache neuägyptischer Erzählungen (1950).

Kang, S.-M., Divine War in the Old Testament and in the Ancient Near East (1989).

Kendall, T., The Origin of the Napatan State: El Kurru and the Evidence for the Royal Ancestors, in: Meroitica 15, 1999, 3-177.

Lange, K., Sesostris. Ein ägyptischer König in Mythos, Geschichte und Kunst (1954).

Lübbe, H., Zur Identitätspräsentationsfunktion der Historie, in: O. Marquard/K. H. Stierle (Hgg.), Identität (1979), 277-292.

Maschinist, P., The Assyrians and their Babylonian Problem, in: Jahrbuch des Wissenschaftskollegs zu Berlin 1984/85, 1985, 353-364.

Neumann, M. (Hg.), Erzählte Identitäten (2000).

Posener, G., Littérature et politique dans l'Égypte de la XIIe dynastie (1956).

Török, L., The Birth of an African Kingdom, Kush and Her Myth of the State in the First Millennium BC (1995).

Ders., The Kingdom of Kush. Handbook of the Napatean-Meoritic Civilization (1997).

Ders., The Image of the Ordered World in Ancient Nubian Art. The Construction of the Kushite Mind, 800BC-300AD (2002), 368-398.

Verhoeven, U., Ein historischer "Sitz im Leben" für die Erzählung von Horus und Seth des Papyrus Chester Beatty I, in: M. Schade-Busch (Hg.), Wege öffnen. Festschrift für Rolf Gundlach zum 65. Geburtstag (1996), 347-363.

Way, T. v. d., Die Textüberlieferung Ramses' II. zur Qades-Schlacht. Analyse und Struktur (1984).

Ders., Göttergericht und „heiliger Krieg“ im Alten Ägypten. Die Inschriften des Merenptah zum Libyerkrieg des Jabres 5 (1992).

Weinrich, H., Tempus - Besprochene und erzählte Welt (1964).

White, H. V., Metahistory: The Historical Imagination in Nineteenth Century Europe (1973). dt. Metahistory: die historische Einbildungskraft im 19. Jahrhundert in Europa (1991).

Yoyotte, J., Notes et documents pour servir à l'histoire de Tanis I. - Bocchoris à Tanis et l'expansion des premiers rois saïtes vers l'Orient, in: Kêmi 21, 1971, 35- 45.

Zandee, J., Le messie, in: Revue de l'Histoire des Religions 180, 1971, 3-28. 